\title{
Uma Abordagem de Gerenciamento de Conflitos Metacognitivos Sensível a Contexto para Sistemas Colaborativos
}

\section{Alternative Title: A Context-sensitive Metacognitive Conflict Management Approach for Collaborative Systems}

\author{
Heremita Brasileiro Lira \\ Instituto Federal de Educação, Ciência e Tecnologia \\ da Paraíba - IFPB, Unidade Acadêmica Informática \\ Avenida Primeiro de Maio, 720 \\ João Pessoa-PB, Brasil \\ heremita@ifpb.edu.br
}

\author{
Patricia Azevedo Tedesco \\ Universidade Federal de Pernambuco - UFPE, \\ Centro de Informática - CIN \\ Cidade Universitária, 50740-540 \\ Recife-PE, Brasil \\ pcart@cin.ufpe.br
}

\begin{abstract}
RESUMO
$\mathrm{O}$ advento das organizações virtuais e dispersas aumenta a demanda por sistemas colaborativos e incentiva pesquisas na área de Computer Supported Cooperative Work (CSCW). Os conflitos são inerentes ao trabalho colaborativo e podem motivar a reflexão e interação entre os envolvidos. Entretanto, as abordagens atuais de gerenciamento de conflito não proveem mecanismos para estimular tais processos. Além disso, poucos utilizam o contexto no apoio à resolução do conflito. O objetivo desse trabalho é apresentar uma abordagem de Gerenciamento de Conflitos Metacognitivos Sensível a Contexto (GCSC) para sistemas de planejamento colaborativo que promova a reflexão e interação entre os envolvidos com a finalidade de melhorar a colaboração. A avaliação da abordagem foi realizada através da implementação de um protótipo utilizando ontologia e de um estudo experimental.
\end{abstract}

\section{Palavras-Chave}

Trabalho Colaborativo Apoiado por Computador. Gerenciamento de Conflito. Contexto Computacional. Planejamento Colaborativo.

\begin{abstract}
The advent of virtual and dispersed organizations increases the demand for collaborative systems and encourages research in the area of Computer Supported Cooperative Work (CSCW). Conflicts are inherent to collaborative work and can motivate reflection and interaction among stakeholders. However, current approaches to conflict management does not provide mechanisms to stimulate these processes. Moreover, only few use the context in supporting the resolution of the conflict. The aim of this work is to present a Context Sensitive Metacognitive Conflict Management (GCSC) approach for collaborative planning systems that promote reflection and interaction among stakeholders in order to improve collaboration. The approach evaluation was performed by using a prototype implementation of an ontology and an experimental study.
\end{abstract}

Permission to make digital or hard copies of all or part of this work for personal or classroom use is granted without fee provided that copies are not made or distributed for profit or commercial advantage and that copies bear this notice and the full citation on the first page. To copy otherwise, or republish, to post on servers or to redistribute to lists, requires prior specific permission and/or a fee.

SBSI 2015, May 26-29, 2015, Goiânia, Goiás, Brazil.

Copyright SBC 2015.

\section{Categories and Subject Descriptors}

H.5.3 [Information Interfaces and Presentation]: Group and Organization Interfaces - Collaborative computing, Computersupported cooperative work.

\section{General Terms}

Human Factors; Management; Design.

\section{Keywords}

Collaborative Work Supported by Computer. Conflict Management. Computational Context. Collaborative Planning.

\section{INTRODUÇÃO}

Com o advento das organizações virtuais e dispersas, cresce a demanda de trabalho colaborativo, aumentando a complexidade das tarefas, pois requer a integração de diferentes habilidades para trabalhar conjuntamente em diversas fases de um projeto [21]. Isso tem gerado demandas por sistemas colaborativos e incentivado pesquisas na área de CSCW [8][10]. Os conflitos são inerentes ao trabalho colaborativo [6]. Quando ocorrem em situações de planejamento colaborativo são oportunidades de aprendizado e colaboração [28] e como tal podem ser gerenciados [6][24] para motivar a reflexão e interação entre envolvidos [28].

Entretanto, as abordagens atuais de gerenciamento de conflitos estão voltadas para sua resolução [32] e não proveem mecanismos para mediá-los, de forma a estimular a reflexão e interação entre os envolvidos [28]. Muito embora utilizados como sinônimos, os termos "gerenciamento de conflitos" e a "resolução de conflitos" não o são. A resolução envolve a redução ou eliminação de conflitos. Normalmente, as estratégias de resolução de conflito são voltadas para processos de negociação, mediação ou arbitragem [24]. O gerenciamento é mais amplo e envolve a concepção de estratégias para minimizar as disfunções e melhorar as funções construtivas do conflito com o objetivo de melhorar a aprendizagem e a cooperação. $\mathrm{O}$ gerenciamento de conflitos não implica necessariamente na sua resolução [23].

O objetivo deste trabalho é apresentar uma abordagem de gerenciamento de conflitos metacognitivos sensível a contexto (GCSC), para sistemas de planejamento colaborativo, que incentive a reflexão e interação entre os envolvidos, motivando o processo de colaboração. No planejamento de um projeto, uma 
das principais ferramentas administrativas utilizadas para definir as tarefas necessárias ao projeto é a técnica $5 \mathrm{~W} 2 \mathrm{H}$ [17]. O 5W2H é uma ferramenta simples com capacidade de definir o conteúdo de tarefas, em um plano, respondendo as seguintes questões: $\mathrm{O}$ que? (What?) deverá ser feito; Por quê? (Why) deverá ser feito; Onde? (Where) será feito; Quando? (When) será feito; Quem? (Who?) será o responsável; Como? (How) será feito e Quanto Custa? (How Much?) as tarefas a serem realizadas.

No planejamento colaborativo uma mudança, realizada por um usuário em uma tarefa, pode significar uma alternativa de solução (conflito metacognitivo) e deve ser objeto de reflexão e interação entre os envolvidos na definição da tarefa. Uma mudança em uma tarefa significa mudar algum conteúdo do modelo $5 \mathrm{~W} 2 \mathrm{H}$, que determina as informações necessárias na definição de uma tarefa.

$\mathrm{Na}$ Abordagem de GCSC utilizamos como base os fundamentos relacionados ao conflito metacognitivo definido em [28]. De acordo com [28], o conflito metacognitivo é o conflito relacionado a como construir alternativas para resolver um determinado problema e como escolher entre essas alternativas. Segundo [28], esses conflitos são oportunidades de aprendizado e colaboração e devem ser gerenciados para motivar a reflexão e interação entre os envolvidos no planejamento colaborativo. $\mathrm{O}$ conflito metacognitivo representa a diferença de ponto de vista dos usuários com relação às alternativas de solução para a definição de uma tarefa. Esses conflitos podem ser detectados pela mudança em algum conteúdo da tarefa realizada pelo usuário.

Os fundamentos relacionados ao contexto utilizados nessa abordagem são os definidos por [3] e utilizados por [30]. Para [30], o contexto é um conjunto de informações que pode ser utilizado para caracterizar uma situação em que ocorra interação entre um usuário e uma aplicação. Estes autores fazem uma distinção clara entre dois conceitos: contexto e elemento contextual (EC). Segundo estes autores, "Um elemento contextual (EC) é qualquer dado, informação ou conhecimento que permite caracterizar uma entidade em um domínio. O contexto da interação entre um agente e uma aplicação, para executar alguma tarefa, é o conjunto de elementos contextuais instanciados que são necessários para apoiar a tarefa atual. Um EC é um tipo de informação que pode ser conhecida, codificada e representada antecipadamente; ela é estável e pode ser definida em tempo de projeto. O contexto é dinâmico, depende da tarefa atual do agente e deve ser construído em tempo de execução, quando uma interação ocorre". Neste trabalho o contexto será utilizado para melhorar a percepção e a assistência no gerenciamento de conflitos metacognitivos, ajudando na definição das estratégias de gerenciamento de conflitos.

A metodologia de desenvolvimento deste trabalho pode ser detalhada em quatro etapas. Na primeira etapa realizamos um levantamento do referencial teórico relacionados à Colaboração, Conflitos e Contexto. Em uma segunda etapa executamos a Revisão Sistemática da Literatura de Gerenciamento de Conflitos em Sistemas Colaborativos em CSCW. Na terceira etapa desenvolvemos a Abordagem de GCSC. Por fim, implementamos o protótipo e experimentação para avaliação da abordagem.

\section{TRABALHOS RELACIONADOS}

Esta seção discute os principais resultados da Revisão Sistemática da Literatura (RSL) para identificar os trabalhos relacionados ao gerenciamento de conflitos em sistemas colaborativos na última década. A RSL foi realizada conforme processo definido por [14], seguindo o protocolo descrito em [2].
Um dos principais resultados observados na RSL foi que, $17 \%$ dos estudos selecionados tem como foco a "detecção do conflito", e os $83 \%$ restantes utilizam uma abordagem voltada para o processo de "resolução de conflito", conforme definido na Seção 1 por [24] e [23]. Apesar dos trabalhos de [12], [16] e [20] utilizarem o termo "processo de gerenciamento de conflito", todos têm como foco o processo de resolução de conflito. Nesses estudos as estratégias de resolução de conflitos utilizadas estão voltadas para o que acontece após a ocorrência do conflito.

Uma segunda observação importante é que menos de $10 \%$ dos estudos selecionados utilizam algum tipo de contexto computacional nas estratégias de "resolução". O trabalho [9] fundamenta suas estratégias de resolução de conflito na Teoria da Atividade, utilizando o contexto computacional do ambiente, da interação e da atividade. O estudo [26] propõe um sistema de resolução de conflito que combina a resolução automática (sem intervenção de agente) com a mediada (com intervenção de agente), explorando informações contextuais da aplicação e dos usuários em sistemas para ambientes inteligentes.

No que diz respeito às intervenções permitidas aos usuários, $70 \%$ dos estudos selecionados possibilitam algum tipo de intervenção do usuário na estratégia de "resolução" adotada. Normalmente essa intervenção está relacionada ao feedback ou à escolha de opções na negociação da resolução dos conflitos. Observamos ainda que os trabalhos de [1], [7] e [26], além de permitirem a intervenção do usuário, também utilizam recomendações no apoio ao processo de resolução do conflito. Os estudos restantes utilizam uma abordagem de resolução automática, não possibilitando a intervenção do usuário na resolução dos conflitos.

Em análise comparativa dos trabalhos relacionados com nossa abordagem, consideramos que o principal diferencial entre eles é que, com relação às estratégias de gestão, nossa proposta utiliza uma abordagem voltada para o gerenciamento do conflito, e não para sua resolução. Nosso foco é gerenciar o conflito para que, ao invés de dificultarem o trabalho colaborativo, se tornem oportunidades de aprendizado e refinamento das soluções produzidas, melhorando a colaboração. Para isto definimos o Modelo de Estratégias de GCSC (Seção 3.3).

Um segundo aspecto que diferencia nosso trabalho é a definição do Modelo de Conflito Metacognitivo (Seção 3.1), que categoriza os conflitos relacionados a diferenças de ponto de vista na construção de soluções para um problema. Nos trabalhos [12] e [20], apesar de referenciarem o conflito de ponto de vista, as soluções são voltadas para o conflito de informação.

Com relação à utilização do contexto, fica claro o diferencial de nossa proposta, pois apenas os trabalhos [9] e [26] utilizam o contexto no apoio à resolução do conflito. Em nossa abordagem o contexto é utilizado para melhorar a percepção e assistência no gerenciamento do conflito. Para isto foi definido, na Seção 3.2, o Modelo de Contexto de Gerenciamento de Conflito Metacognitivo que detalha o contexto relevante neste gerenciamento.

Assim como nos trabalhos de [1], [7] e [26], nossa abordagem permite a intervenção dos usuários na escolha das estratégias, bem como apresenta recomendações que melhoram a percepção do conflito e dos envolvidos, dando apoio ao seu gerenciamento.

Por fim, diferentemente do restante dos trabalhos nossa proposta tem como finalidade suprir a ausência de mecanismos para o gerenciamento de conflitos, para promover a reflexão e interação entre os envolvidos, motivando o processo de colaboração. 


\section{VISÃO GERAL}

Uma visão geral da Abordagem de GCSC é apresentada na Figura 3-1. Os usuários interagem (1) com o ambiente de planejamento colaborativo para definir as tarefas em um projeto. $\mathrm{Na}$ definição de uma tarefa, especificamente nos eventos de mudança em algum conteúdo $5 \mathrm{~W} 2 \mathrm{H}$ da tarefa, o módulo de detecção da Abordagem de GCSC detecta essa mudança e captura essas interações (2) e envia as informações relevantes do contexto do conflito para o módulo de análise. No módulo de análise, as informações do contexto do conflito e seus envolvidos são avaliadas e, com base nelas, o módulo de intervenção dispara ações junto aos usuários, apresentando estratégias de gerenciamento (3) de conflito sensível ao contexto. Os usuários decidem que estratégias utilizar, podendo ainda fornecer Feedback (4) das estratégias apresentadas.

A Abordagem de GCSC, mostrado na Figura 3-1, é composto por três módulos principais: Detecção, Análise e Intervenção. Esses módulos utilizam os Modelos de Conflito, Contexto e Estratégias para identificar e categorizar o conflito e respectivo contexto, bem como selecionar as estratégias de gerenciamento de conflitos, adaptando o comportamento da Abordagem de GCSC de acordo com o contexto. Estes elementos estão detalhados a seguir.

\subsection{Modelo de Conflito Metacognitivo}

A Abordagem de GCSC utiliza o Modelo de Conflito Metacognitivo definido nesta seção para categorizar o conflito detectado de acordo com a mudança realizada no conteúdo 5W2H de uma tarefa. [28] classificou os conflitos metacognitivos em conflitos de reflexão, intenção, ordenação e de objetivos. Em análise comparativa do modelo de conflito de [28] com o modelo $5 \mathrm{~W} 2 \mathrm{H}$ utilizado para representar o conteúdo de uma tarefa, observou-se uma lacuna com relação aos elementos "Onde?", "Quem?" e "Quanto custa?" do modelo 5W2H. Para preenchermos esta lacuna, adaptamos o Modelo de Conflito Metacognitivo de [28], incluindo respectivamente os novos conceitos de conflitos metacognitivos de espaço, responsabilidade e custo. Os conflitos metacognitivos são subdivididos nos seguintes tipos de conflito (conflictType):

- Reflector Conflict (conflictType=what) - o conflito de reflexão está relacionado com a diferença de ponto de vista de como definir as melhores alternativas de solução para um problema e como escolher entre elas. Essas alternativas dizem respeito à reflexão na definição de "O que" a tarefa deve realizar. Ex.: Título da tarefa, categoria (requisito de domínio/contexto).
- Intention Conflict (conflictType=how) - o conflito de intenção refere-se à diferença de ponto de vista quanto aos passos que compõem a alternativa de solução. Ocorrem quando existe diferença na definição de "Como" uma tarefa deve ser realizada, quais os passos necessários à tarefa. Ex.: Qual o processo (clássico/ágil). Como modelar (UML/SPEM).

- Ordering Conflict (conflictType=when) - o conflito de ordenação é aquele que acontece quando existe diferença de ponto de vista na forma de organizar a sequência dos passos definidos para a tarefa. Está relacionado à definição do "Quando" a tarefa deve ser realizada. Ex.: data de início/fim, dias/semanas.

- Goal Conflict (conflictType=why) - o conflito de objetivo refere-se à diferença de ponto de vista nos objetivos do grupo e podem ser subdivididos em: diferenças na definição e no atendimento dos objetivos. Acontecem na reflexão da definição do "Porque" uma tarefa deve ser realizada. Ex.: Atividade Superior/Estrutura Analítica do Projeto (EAP).

- Space Conflict (conflictType=where) - o conflito de espaço (local/tempo) ocorre quando existe diferença de ponto de vista quanto ao espaço "onde" a tarefa deve ser realizada. Ex.: Presencial/Virtual, Síncrono/Assíncrono, Ferramenta.

- Responsibility Conflict (conflictType $=$ who) $-\mathrm{o}$ conflito de responsabilidade ocorre quando existe diferença de ponto de vista com relação à definição do responsável pela tarefa. Refere-se à definição de "Quem" deverá executar a tarefa. Ex.: Desenvolvedor/Design Gráfico, João/Maria.

- Cost Conflict (conflictType=howMuch) - o conflito de custo acontece quando existe diferença de ponto de vista na definição do componente de custo da tarefa. Está relacionado ao "Quanto" deve ser o custo para a realização da tarefa. Ex.: hora/tarefa/diária, estimativa análoga/paramétrica, valor x/y.

\subsection{Modelo de Contexto de Conflito}

Para definir o Modelo de Contexto de Conflito foi utilizado como referência o Metamodelo de Contexto apresentado em [30]. O Modelo de Contexto de Conflito, mostrado na Figura 3-2, descreve as entidades contextuais e os respectivos elementos contextuais (EC), que têm como objetivo caracterizar o contexto relevante do conflito e dos envolvidos no conflito, a serem utilizados pela Abordagem de GCSC.

Tomando como base a visão geral da Abordagem de GCSC, visto na Figura 3-1, a principal atividade realizada pelo usuário no planejamento colaborativo é a definição de tarefas.

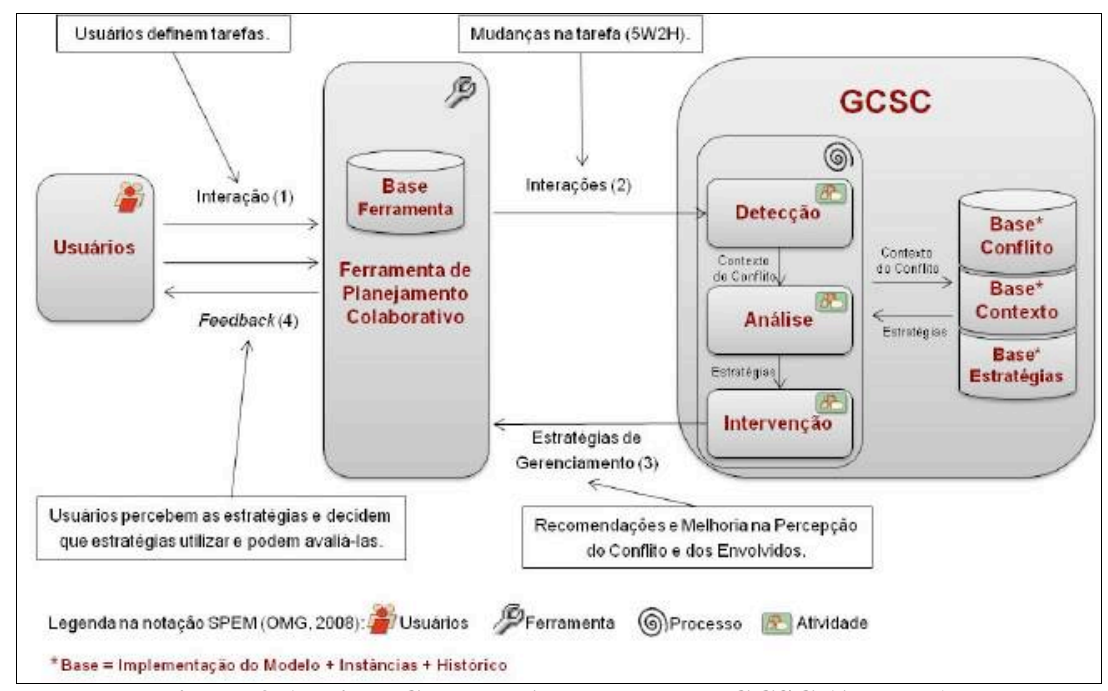

Figura 3-1: Visão Geral da Abordagem de GCSC (Autoras). 
Considerando as mudanças (Change) realizadas pelo usuário em uma tarefa como foco (Focus) principal do contexto relevante para o GCSC, as principais entidades contextuais consideradas são: Conflito (Conflict), Usuários (User) e Tarefa (Task). Essas entidades e respectivos EC definem o Modelo de Contexto de Conflito mostrado na Figura 3-2 e descritos a seguir.

Alguns dos EC relevantes utilizados para caracterizar a entidade contextual Conflito (Conflict) são:

- hasConflictType - caracteriza "o que" está em conflito e indica qual o conteúdo $5 \mathrm{~W} 2 \mathrm{H}$ da tarefa que foi modificado. Permite categorizar o tipo de conflito (conflictType) com base no Modelo de Conflito Metacognitivo. Ex. Conflito de Custo, quando o conteúdo custo (How Much) foi mudado na definição da tarefa.

- hasEnvolved - identifica quem são os usuários que fazem parte do grupo (Group) de envolvidos no conflito. Isto permite utilizar seus EC para motivar a reflexão e interação. Estes usuários serão listados nas notificações e recomendações.

Para caracterizar a entidade contextual Usuário (User), alguns dos EC relevantes utilizados são:

- isAvailable - define a disponibilidade do usuário e indica se o usuário está ocupado, ausente ou disponível no ambiente colaborativo. Este EC poderá ser usado para priorizar a lista de envolvidos. Ex. usuários disponíveis online no ambiente são priorizados quando mostrados os envolvidos no conflito.

- isLocatedIn - indica a localização física do usuário. Usuários mais próximos ou no mesmo local podem interagir face a face, facilitando a reflexão. Ex. Se usuários no escritório, então recomendar reunião presencial.

- hasContactInfo - informa como um usuário pode ser contatado (email, skype, sms, whatsapp, telefone). Permite escolher a melhor forma de entrar em contato, imediatamente (comunicação síncrona) ou em outro momento (assíncrona).

- hasExpertise - indica a expertise de um usuário em um assunto relacionado ao objeto em conflito na tarefa $(5 \mathrm{~W} 2 \mathrm{H})$. Utilizado para priorizar a relação dos envolvidos no conflito.
Alguns dos EC relevantes utilizados para caracterizar a entidade contextual Tarefa (Task) são:

- hasStatus - identifica o status de uma tarefa e identifica o período em que uma tarefa está em fase de planejamento. Os conflitos metacognitivos serão gerenciados apenas nesta fase.

- hasCreator - define quem é o usuário que criou a tarefa. Esse usuário pode fazer parte do grupo de envolvidos no conflito.

\subsection{Modelo de Estratégias de GCSC}

$\mathrm{Na}$ Abordagem de GCSC, o contexto é utilizado para determinar as estratégias de gerenciamento do conflito, que tem como objetivo motivar a reflexão e interação dos envolvidos no planejamento colaborativo. Portanto, o Modelo de Estratégias de GCSC é representado pelo conjunto de estratégias que consideram o contexto e determinam o comportamento da Abordagem de GCSC, melhorando a percepção e assistência no apoio ao gerenciamento do conflito no planejamento colaborativo.

Descrevemos a seguir um subconjunto de estratégias do Modelo de Estratégias de GCSC, que consideram o contexto e determinam o comportamento da abordagem de GCSC:

- NotifyConflict - Quando ocorre um conflito, notificar o conflito aos envolvidos no planejamento, informando "o que" foi mudado na tarefa e o tipo de conflito (has ConflictType), "quem" mudou (hasAgent), "quando" (hasWhen) e "por que" (hasWhy). Ex. "Ocorreu um conflito de custo na tarefa X. João mudou o custo da tarefa de A para B, em 25/07/14 15hs, sugerindo como alternativa considerar o valor hora/trabalho do responsável.

- ExplainImpact - Quando ocorre um conflito, solicitar ao envolvido que mudou (hasAgent) a tarefa para explicar o impacto de sua sugestão. A explicação do impacto será categorizada como alto, médio e baixo impacto.

- ShowEnvolved - Ao notificar um conflito, mostrar a lista de envolvidos (hasEnvolved) com respectivas disponibilidades (isAvailable) (presente ou ausente no ambiente) e expertises (hasExpertise) relacionadas ao tipo de conflito (expert em custo).

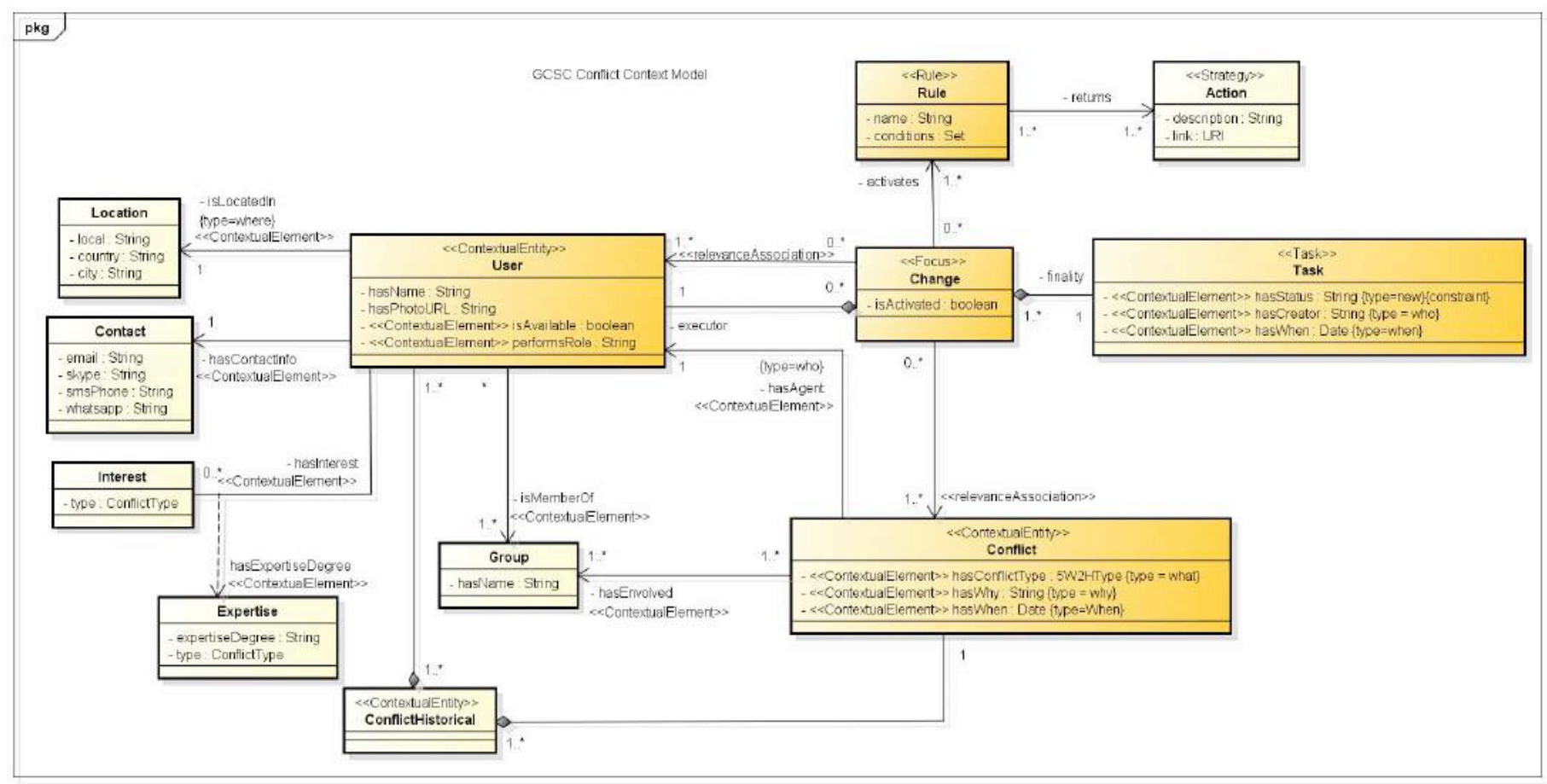

Figura 3-2: Modelo de Contexto de Conflito (Autoras). 
Desta forma, os envolvidos decidem com quem e como interagir, de acordo com seu perfil e dos demais. Ex. Lista de envolvidos: 1) Paulo, online, alta expertise em custo; 2) Lucia, online, baixa expertise em custo; 3 ) Maria, offline, média expertise em tempo.

- FaceMeeting - Se os envolvidos no planejamento estiverem (isLocatedIn) fisicamente no mesmo local, recomendar uma reunião presencial para refletirem sobre os conflitos notificados e categorizados como de alto impacto.

- VirtualMeeting - Se os envolvidos no planejamento estiverem virtualmente disponíveis (isAvailable) no ambiente de gerenciamento do projeto, recomendar reunião virtual para refletirem sobre os conflitos notificados como de alto impacto.

- CheckPortfolio - Se o conflito for categorizado como de alto impacto, recomendar aos envolvidos checar o portfólio (baseline, padrões) relacionado ao tipo de conflito notificado.

- MultipleConflicts - Se em uma única tarefa ocorrerem vários conflitos, fazer a seguinte recomendação aos envolvidos no planejamento: "Atenção, esta tarefa tem vários conflitos, sugerimos uma reunião para reflexão e discussão sobre eles!".

- ConsultExpert - Se um mesmo tipo de conflito se repete várias vezes, na mesma tarefa ou em tarefas diferentes, recomendar ao grupo consultar um expert no assunto relacionado ao tipo de conflito, para ajudar na reflexão ou treinamento.

Este conjunto de estratégias é representado por um conjunto de regras contextuais, que são regras de produção que utilizam os elementos contextuais (EC), e que serão processadas utilizando um motor de inferência. Uma regra de produção é uma declaração lógica de programação que especifica a execução de uma ou mais ações no caso em que as condições definidas forem satisfeitas [25]. Um exemplo de uma regra é apresentada na Seção 3.7.

\subsection{Módulo de Detecção do Conflito}

A detecção do conflito é realizada a partir dos eventos de mudança em algum conteúdo da tarefa $(5 \mathrm{~W} 2 \mathrm{H})$, efetuada por um usuário na atividade de definição de uma tarefa. Por exemplo, um usuário na definição de uma tarefa muda o custo que o próprio usuário ou outro havia definido para a tarefa. Esses eventos de mudança devem ser motivo para reflexão e interação entre os envolvidos no conflito, a fim de entender os diferentes pontos de vista na definição da tarefa, possibilitando uma solução mais elaborada. Após a detecção, o conflito é categorizado de acordo com o Modelo de Conflito Metacognitivo, definido na Seção 3.1, para que então seja realizada a aquisição do contexto.

Para a aquisição do contexto, foram identificadas duas fontes de contexto: (1) a base de dados do ambiente de planejamento colaborativo para detecção dos eventos de mudanças (5W2H) nas tarefas; (2) as bases de dados da nossa abordagem com respectivas bases históricas que representam os Modelos de Conflito, Contexto e Estratégias da Abordagem de GCSC, definidas nas seções anteriores. Nessas fontes serão adquiridos os EC, como definidos no Modelo de Contexto do Conflito. Essas informações são enviadas para o módulo de análise do conflito.

\subsection{Módulo de Análise do Conflito}

O módulo de análise tem como entrada o contexto do conflito e dos envolvidos. Esse módulo é responsável pela análise do contexto e pelo processamento das regras contextuais, com base nos contextos do conflito e dos envolvidos recebidos do módulo de detecção. O processamento das regras contextuais é realizado para identificar as estratégias de gerenciamento do conflito que serão enviadas para o módulo de intervenção. Essas estratégias determinam o comportamento do GCSC. Para esse processamento é utilizado o Modelo de Contexto do Conflito, detalhado na Figura 3-2, e o Modelo de Estratégias, mostrado na Seção 3.3.

No processamento das regras contextuais são avaliadas as condições de cada regra, considerando o contexto, e são identificadas as respectivas ações de cada regra que são as estratégias de gerenciamento do conflito selecionadas. Estas estratégias são enviadas para o módulo de intervenção.

\subsection{Módulo de Intervenção no Conflito}

Esse módulo é responsável pela apresentação das estratégias de GCSC provendo os serviços sensíveis ao contexto, como assistência e melhoria na percepção do conflito e no seu gerenciamento. Os serviços de assistência no GCSC são disseminados por meio das recomendações disparadas pelas estratégias de gerenciamento que incentivam a reflexão e interação no conflito. Essas recomendações são apresentadas como mensagens temporárias na interface dos envolvidos e serão anexadas ao histórico do conflito na tarefa.

Os serviços de melhoria na percepção no GCSC são disseminados mediante notificação do conflito (interface dos envolvidos, tarefa, email). No histórico do conflito na tarefa, sempre que um conflito for acessado, os elementos contextuais do conflito e dos envolvidos serão apresentados em detalhe, ampliando-se a percepção e permitindo aos envolvidos decidir a melhor forma de interagir de acordo com o contexto. O módulo de intervenção tem como saída a apresentação das estratégias de gerenciamento do conflito no ambiente de planejamento colaborativo, adaptando o comportamento do GCSC de acordo com o contexto.

\subsection{Cenário de Conflito}

Para exemplificar a aplicação da Abordagem de GCSC descrevemos a seguir um cenário de conflito metacognitivo no planejamento colaborativo. João, gerente de custo de um projeto colaborativo, está no escritório do projeto acessando o ambiente de planejamento colaborativo e muda o custo de uma tarefa, que foi criada por Maria, gerente de tempo do projeto.

Sem considerar a aplicação da Abordagem de GCSC para este cenário, o que normalmente aconteceria é que os demais envolvidos no planejamento colaborativo, incluindo Maria, não perceberiam esta mudança e nem teriam a oportunidade de entender o porquê João a realizou. Provavelmente, esta mudança só seria percebida em um momento posterior, talvez até depois da fase de planejamento, acarretando retrabalho ou um mal estar entre os envolvidos, por não terem participado da mudança.

Considerando o cenário apresentado anteriormente, mostramos a seguir a aplicação da Abordagem de GCSC. Inicialmente, o Módulo de Detecção identifica o evento de mudança no custo da tarefa e identifica o tipo de conflito de acordo com o Modelo de Conflito detalhado na Seção 3.1. Como a mudança na tarefa foi realizada no conteúdo de custo da tarefa (howMuch do $5 \mathrm{~W} 2 \mathrm{H}$ ), este evento será categorizado como um conflito de custo (CostConflict). Os envolvidos no conflito são: João, que está definindo a tarefa e mudou seu custo; Maria, que criou a tarefa; Lucia, que é membro do grupo de planejamento juntamente com João e Maria; Paulo, que é consultor de custos do projeto.

O Quadro 3-1 detalha as instâncias da entidade contextual Usuários (User) envolvidos no conflito. O contexto identificado no Módulo de Detecção é enviado para o Módulo de Análise para processamento das regras contextuais, de acordo com o Modelo de Estratégias de GCSC. Vários outros cenários foram definidos e utilizados na avaliação da abordagem. 
Quadro 3-1: Exemplo de instâncias da classe User.

\begin{tabular}{|l|l|l|l|l|}
\hline $\begin{array}{c}\text { Elementos } \\
\text { Contextuais }\end{array}$ & \multicolumn{1}{|c|}{ João } & \multicolumn{1}{|c|}{ Maria } & \multicolumn{1}{|c|}{ Lucia } & \multicolumn{1}{|c|}{ Paulo } \\
\hline isAvailable & Sim & Não & Sim & Sim \\
\hline performsRole & Ger. Custo & Ger. Tempo & Ger. Escopo & Consultor \\
\hline isLocatedIn & Escritório & Escritório & Escritório & Escritório \\
\hline hasContactInfo & Email & Email & Email & Email \\
\hline hasInterest & Custo & Tempo & Escopo & Custo \\
\hline hasExpertise & Médio & Médio & Baixo & Alto \\
\hline isMemberOf & Planejamento & Planejamento & Planejamento & Execução \\
\hline
\end{tabular}

Para ilustrar o uso do Modelo de Estratégias de GCSC, apresentamos a regra contextual e respectivas estratégias de GCSC considerando o cenário apresentado:

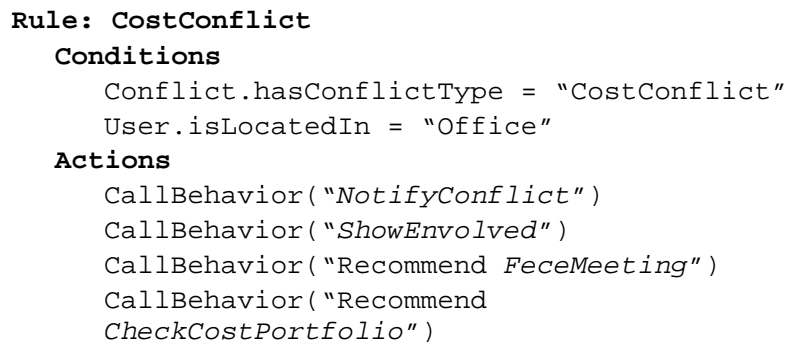

Percebendo essas informações, os envolvidos podem decidir com quem, quando e como interagir, de acordo com o próprio contexto do conflito e dos demais envolvidos.

\section{AVALIAÇÃO}

A avaliação da Abordagem de GCSC foi realizada em duas etapas. Na primeira foi implementado um protótipo da abordagem utilizando ontologia como prova de conceitos [4]. Na segunda foi realizado um estudo experimental [29] para avaliar, do ponto de vista de usuários com experiência no planejamento colaborativo, se as estratégias de gerenciamento de conflitos definidas na abordagem, incentivam a reflexão e interação entre os envolvidos.

\subsection{Protótipo da Abordagem de GCSC}

O processo de construção da ontologia de GCSC é o descrito em [11] e [18] para o Protégé [22]. Para implementar os conceitos da ontologia utilizamos a linguagem axiomática OWL-DL $(O W L-$ Description Logics) [31]. Para a validação dos conceitos utilizamos o motor de inferência Pellet [27], instalado como plugin no Protégé. Na Figura 4-1 foram omitidas relações para facilitar a visualização no OntoGraf [22].

Como mostra a Figura 4-1, os conceitos que fazem parte da Abordagem de GCSC estão relacionados ao conceito principal de GCSC. Inicialmente, foram implementados os conceitos de conflito (Conflict) e de usuários (User) envolvidos no conflito, que possuem (has) elementos contextuais que caracterizam o contexto relevante para seu gerenciamento. A descrição detalhada de cada conceito pode ser revista na Seção 3, que define os principais elementos da Abordagerm de GCSC.

$\mathrm{Na}$ validação da ontologia vários cenários instanciados foram executados e validados de acordo com os conceitos implementados na ontologia. Utilizamos como exemplo o cenário de conflito descrito na Seção 3.7, e instânciado na Figura 4-1, que mostra o conflito (Conflit1) sinalizado pela mudança no custo (cost) de uma tarefa (Task) realizado por John (User). Os envolvidos (User) no conflito que fazem parte do grupo (Group) de planejamento são: Lucy, Mary, Paul e John.

Para implementar e validar as estratégias na ontologia utilizamos ainda os seguintes recursos: o Plugin SWRLDroolsTab e o Rule Engine Drools, ambos registrados no SWRLTab [19] do Protégé. A SWRL (Semantic Web Rule Language) [12] estende a OWL e é uma linguagem para descrição de regras usando RuleML e OWL. O SWRLDroolsTab faz o mapeamento de axiomas OWL2 estendido com SWRL para regras Drools [19]. O objetivo do mapeamento é executar as regras e garantir a consistência da ontologia.

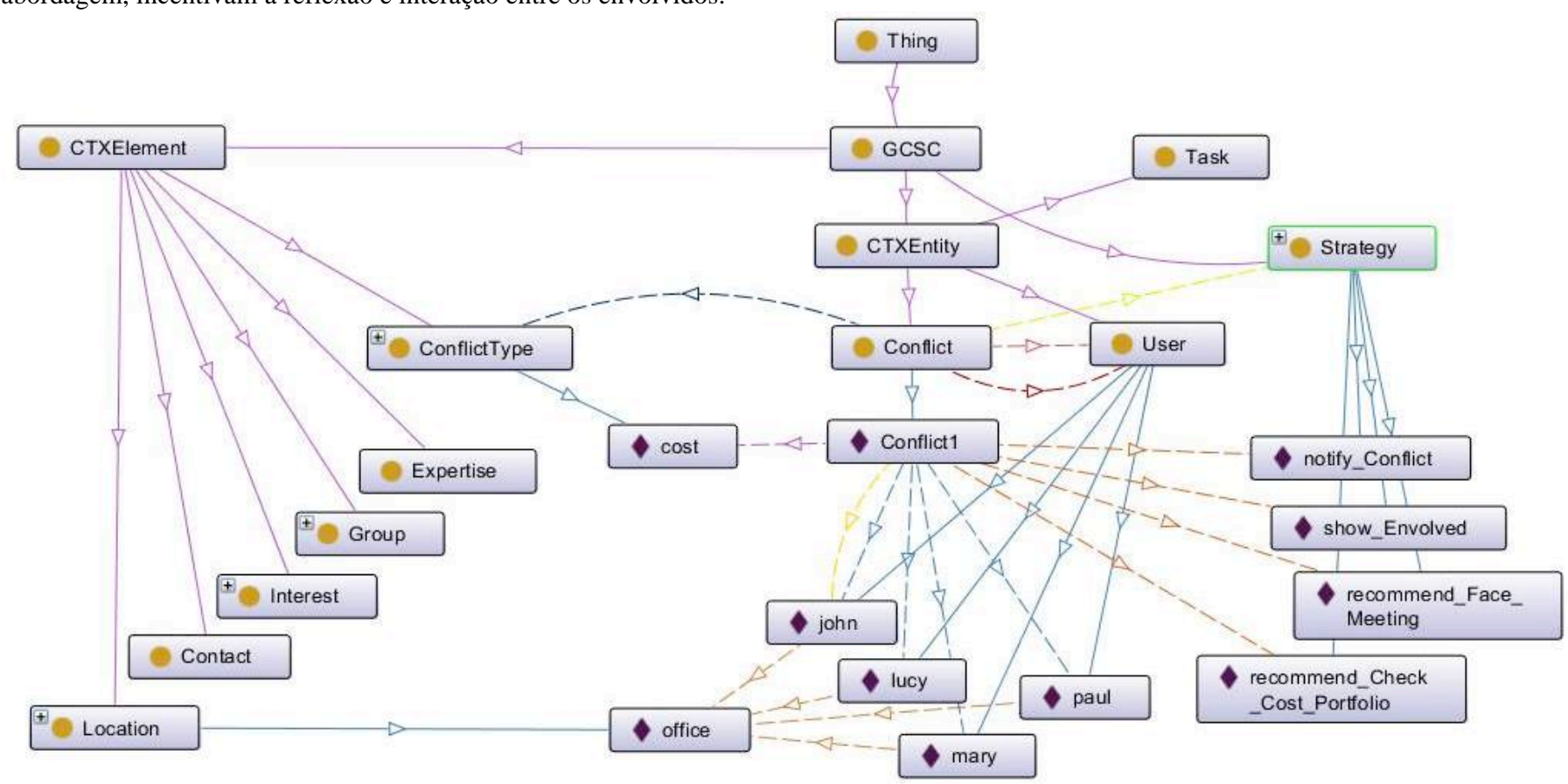

Figura 4-1: Visão das estratégias (Strategy) disparadas (Autoras). 
Foram implementadas as estratégias definidas na Seção 3.3. O Quadro 4-1 mostra uma visão das estratégias disparadas de acordo com o contexto do conflito e dos envolvidos, no cenário considerado. Utilizamos a notação do Editor de Regras SWRL Rules do Protégé, de fácil leitura por humanos.

Quadro 4-1: Visão das estratégias ativadas conforme cenário.

\begin{tabular}{|c|c|}
\hline $\begin{array}{r}\text { Iden } \\
\text { das E }\end{array}$ & $\begin{array}{r}\text { Estr } \\
\text { (para cada contextc }\end{array}$ \\
\hline Noti & $\begin{array}{ll}\text { Conflict(?x) } & \rightarrow \\
\text { notify_Conflict) }\end{array}$ \\
\hline Shon & $\begin{array}{l}\text { Conflict(?x) } \\
\text { show_Envolved) }\end{array} \rightarrow$ \\
\hline RecCost & $\begin{array}{l}\text { Conflict(?x) } \wedge \text { hasC } \\
\rightarrow \text { activateStrategy(?x, rec }\end{array}$ \\
\hline Face & $\begin{array}{l}\text { Conflict(?x) } \wedge \text { User(?y) } \wedge \text { isLocatedIn(?y, } \\
? \mathrm{z}) \rightarrow \text { activateStrategy(?x, recommendFaceMeeting }\end{array}$ \\
\hline
\end{tabular}

Para a validação das estratégias, na segunda fase da validação, utilizamos o motor de regras Drools, onde foram instanciados os cenários e inferidas as regras que implementam as estratégias de gerenciamento de conflitos de acordo com o contexto dos cenários. Como pode ser observado na Figura 4-1, as estratégias "NotifyConflict", "ShowEnvolved", "RecommendFaceMeeting" e "RecommendVerifyCostPortfolio", são disparadas considerando o contexto do conflito e dos envolvidos no planejamento.

Para cada novo conceito ou estratégia implementada na ontologia de GCSC foram realizados todos os procedimentos para validação da ontologia e os resultados foram positivos, ou seja, a verificação da consistência, a classificação da taxonomia, a inferência de tipos e a execução de testes da ontologia não apresentaram erros com a inclusão de novos conceitos e estratégias.

\subsection{Estudo Experimental}

Para a experimentação foi utilizado o trabalho de [29] que define o processo para a organização de estudos experimentais. $O$ objetivo é verificar se a abordagem oferece estratégias de gerenciamento de conflitos que incentivam a reflexão e interação entre os envolvidos, do ponto de vista de usuário com experiência no planejamento colaborativo.

Foram considerados 51 (cinquenta e um) indivíduos que participaram do experimento de forma voluntária e aleatória, respondendo a um questionário enviado por email, para listas de alunos e profissionais, de universidade e empresas, com experiência no planejamento colaborativo em projetos de desenvolvimento de software colaborativos e distribuídos. O perfil dos participantes foi identificado como sendo: $60 \%$ com mais de 30 (trinta) anos, $75 \%$ com formação na área de Ciência da Computação, $67 \%$ com pós-graduação, $61 \%$ com mais de 2 (dois) anos de experiência e $18 \%$ com mais de 10 (dez) anos de experiência.

As questões avaliadas dizem respeito às 12 (doze) estratégias de gerenciamento de conflito definidas no Modelo de Estratégias de GCSC. Estas questões foram detalhadas no questionário aplicado no experimento. Para cada questão os participantes avaliaram as estratégias, escolhendo entre as respostas: Concordo Plenamente (CP), Concordo (C), Neutro (N), Discordo (D) e Discordo Plenamente (DP).

A métrica definida para medir os resultados da avaliação dos participantes é o Índice de Concordância por participante $\left(I C_{i}\right)$, uma medida para identificar o grau de concordância dos participantes com relação às estratégias apresentadas. Este índice foi definido com base no índice de concordância Kappa [5], e mede o grau de concordância além do que seria esperado tão somente pelo acaso. Com os IC por participantes, calculamos o valor médio do Índice de Concordância Geral $\overline{I C}$.

Para avaliar se a medida obtida no nosso indicador de concordância é satisfatória ou não, utilizamos como base o trabalho de [15], sugerindo a seguinte interpretação: se o Índice de Concordância Geral (IC) for maior ou igual a 0.8 , consideramos que a medida é satisfatória. A coleta dos dados foi realizada através do envio de email com o questionário de avaliação para os participantes, de maneira que não causasse nenhum efeito significativo ao objeto (estratégias) que está sendo estudado.

Utilizando as métricas definidas anteriormente, para cada conjunto de respostas de um participante foi calculado o $I C_{i}$ e o Índice de Concordância Geral ( $\overline{\mathrm{IC}})$, tendo como resultado final o $\overline{\mathrm{IC}}=0.92$. Os resultados dos $I C_{i}$ por participante e do $\overline{\mathrm{IC}}$ podem ser observados na Figura 4-2. Como o IC é uma medida de tendência central, esta média foi calculada considerando o intervalo de confiança entre [0.87, 0.97], para um nível de confiança de $95 \%$.

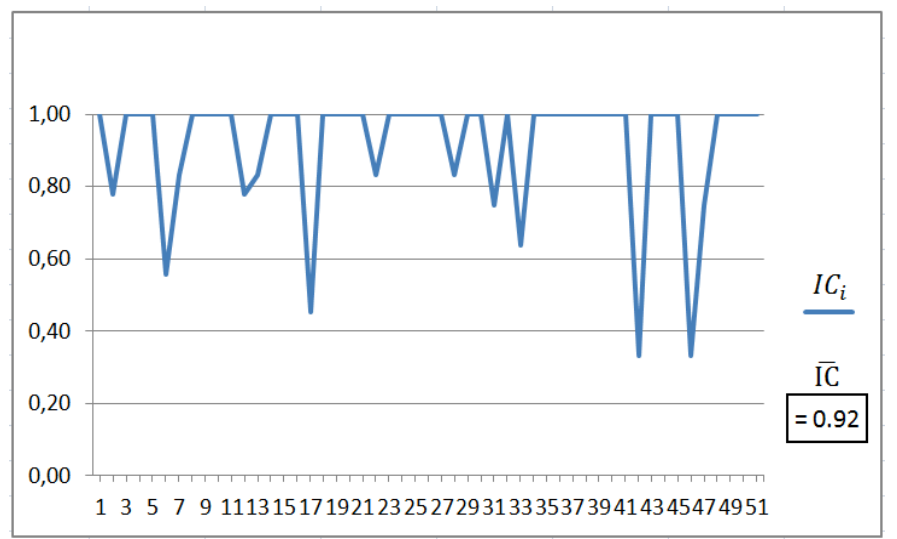

Figura 4-2: Os resultados dos ICi e do IC

Considerando os resultados e as métricas definidas, podemos realizar a seguinte verificação: o $\overline{\mathrm{IC}}=0.92$ está dentro do intervalo de $[0.8,1]$. Podemos então afirmar que, considerando o cenário avaliado e o resultado da métrica utilizada, temos indícios que as estratégias de gerenciamento de conflito incentivam a reflexão e interação entre os envolvidos no planejamento colaborativo.

\section{CONCLUSÃO}

Nosso trabalho de pesquisa tem como foco os conflitos metacognitivos, que estão relacionados ao confronto de ideias no trabalho colaborativo. Estes conflitos podem ser gerenciado de forma criativa para motivar a colaboração. $\mathrm{O}$ uso de contexto é essencial no gerenciamento de atividades colaborativas, pois o entendimento compartilhado das divergências e soluções resulta na sensação de que todos podem contribuir e sair ganhando.

A análise dos resultados da avaliação da abordagem indicam que, considerando os cenários e métricas utilizadas, temos indícios de que a Abordagem de GCSC é viável computacionalmente e que suas estratégias de gerenciamento de conflito incentivam a reflexão e interação entre os envolvidos no planejamento colaborativo, motivando a colaboração.

As principais contribuições deste trabalho são: a Revisão Sistemática da Literatura; a Especificação da Abordagem de GCSC com seus conceitos, modelos e estratégias; o Protótipo da 
Abordagem de GCSC e o Estudo Experimental. Estas contribuições compõem uma visão geral do gerenciamento de conflito em sistemas colaborativos e podem ser utilizados por pesquisadores e profissionais de áreas como CSCW, IHC, Contexto e Inteligência Computacional.

As contribuições e limitações deste trabalho abrem perspectivas de trabalhos futuros, tais como: estender o Modelo de Estratégias de GCSC, ampliar os testes do protótipo com cenário mais complexos e desenvolver um plugin da Abordagem de GCSC.

\section{REFERÊNCIAS}

[1] Aiken, R. M. et al. 2005. Interaction and Collaboration using an Intelligent Collaborative Learning Environment. Revue Education and Information Technologies, v. 10, p. 65-80.

[2] Biolchini, J. et al. 2005. A Systematic Review Process to Software Engineering. In: ESELAW05 - Experimental Software Engineering Latin American Workshop, Uberlandia. Proceedings of the ESELAW05, v. 1.

[3] Brézillon, P. 2007. Context modeling: Task model and model of practices. In: Proc. of the 6th International and Interdisciplinary Conference on Modeling and Using Context (CONTEXT'07), LNAI 4635, p. 122-135, Roskilde.

[4] Carsten, B. 1989. Proof of concept prototype. In: Power Conversion and Intelligent Motion Magazine. Carsten's Corner column, p. 38.

[5] Cohen, J. 1968. Weighted kappa: Nominal scale agreement provision for scaled disagreement or partial credit. Psychological Bulletin, v. 70, n. 4 p. 213-20.

[6] De Dreu, C. K. W.; Gelfand, M. J. 2013. The Psychology of Conflict and Conflict Management Organizations. SIOP Organizational Frontiers Series. Psychology Press; Reprint edition (Jan, 2013).

[7] Dewan, P.; Hegde, R. 2007. Semi-synchronous conflict detection and resolution in asynchronous software development. ECSCW 2007, p. 159-178.

[8] Ellis, C.A. et al. 1991. Groupware - Some Issues and Experiences. Communications of the ACM, v. 34.

[9] Fuentes, R. et al. 2005. Managing Conflicts between Individuals and Societies in Multi-Agent Systems. In: 5th International Workshop Engineering Societies in the Agents World.

[10] Grudin, J.; Poole, E. S. 2010. Wikis at work: success factors and challenges for sustainability of enterprise wikis. In Proceedings of the 6th International Symposium on Wikis and Open Collaboration, ACM, NY, USA, v. 5, p. 1-5.

[11] Horridge, M.; Brandt, S. A 2011. Practical Guide To Building OWL Ontologies Using Protégé 4 and CO-ODE Tools. Edition 1.3. The University of Manchester.

[12] Horrocks, I. et al. 2004. SWRL: A Semantic Web Rule Language Combining OWL and RuleML. W3C Member Submission. Disponível em: <http://www.w3.org/Submission /2004/SUBM-SWRL-20040521> Acesso em 06 ago. 2014.

[13] Jiao, Y. et al. 2005. Towards a Systematic Conflict Resolution Policy in Multi-agent System: A Conceptual Framework. In Proceedings of CSCWD 2005, p. 274-283.

[14] Kitchenham, B. 2004. Procedures for Performing Systematic Reviews. TR/SE-0401, Department of Computer Science, Keele University and National ICT, Australia.
[15] Landis, J. R; Koch, G. G. 1977. The measurement of observer agrément for categorical data. Biometrics, v.33.

[16] Ma, J. et al. 2008. Conflict resolution for collaborative design based on rough set theory. Computer Supported Cooperative Work in Design, 2008. CSCWD 2008. 12th International Conference, p.64-69.

[17] Meireles, M. 2001. Ferramentas administrativas para planejar contramedidas e comunicar. Série: Excelência empresarial, v.3. São Paulo: Arte \& Ciência.

[18] Noy, N. F.; Mcguinness, D. L. 2001. Ontology Development 101: A Guide to Creating Your First Ontology. Stanford University, Stanford, CA.

[19] O'Connor, M.J. et al. 2005. Supporting Rule System Interoperability on the Semantic Web with SWRL. In: 4th International Semantic Web Conference (ISWC), Galway, Ireland, Springer Verlag, LNCS 3729, p. 974-986.

[20] Ouertani, M. 2008. Supporting conflict management in collaborative design: An approach to assess engineering change impacts. Computers in Industry v. 59, n. 9.

[21] Phillips, S. et al. 2014. Understanding and Improving Software Build Teams. In Proceedings of the 36th International Conference on Software Engineering, ACM.

[22] PROTÉGÉ. 2012. Protégé Community of Practice (CoP), Disponível em: <http://protege.cim3.net/cgi-bin/wiki.pl> Acesso em: 01 out. 2014.

[23] Rahim, M. A. 2002. Toward a theory of Managing Organizational Conflict. In TheInternational Journal of Conflict Management, v. 13, n. 3, p. 206-235.

[24] Robbins, S. P. 2014. Fundamentos do Comportamento Organizacional. 12. ed., Pearson Prentice Hall, SP.

[25] Russel, S., Norvig, P. 2003. Artificial Intelligence: A Modern Approach. Prentice Hall.

[26] Shin, C. et al. 2008. Mixed-Initiative conflict resolution for Context-aware applications. Proc. of International Conference on Ubiquitous Computing, p. 262-271.

[27] Sirin, E. et al. 2007. Pellet: A practical OWL-DL Reasoner. Web Semantics: Science, Services and Agents on the World Wide Web, v. 5, n. 2, p. 51-53.

[28] Tedesco, P. A. 2003. MArCo: Building an Artificial Conflict Mediator to Support Group Planning Interactions. International Journal of Artificial Intelligence in Education, 13 IOS press, p. 117-155.

[29] Travassos, G. et al. 2002. Introdução à Engenharia de Software Experimental. ES590/02-Apr., COPPE/UFRJ.

[30] Vieira, V. et al. 2011. Designing context-sensitive systems: An integrated approach. Expert Systems with Applications, v. 38 , n. 2, p. 1119-1138.

[31] W3C. 2012. OWL 2 Web Ontology Language, Document Overview (Second Edition). W3C Recommendation, December 2012.Dispinível em: <http://www.w3c.org /TR/owl2-overview>. Acesso em 01 out. 2014.

[32] Zhang, G.,; Purao, S. 2014. CM2: A Case-Based Conflict Management System. Proceedings of the Conference on Design Science in Information Systems and Technologie. Miami, FL. Jun., LNCS 8463, p. 257-272. 